\title{
DỨcin
}

Technological University Dublin ARROW@TU Dublin

\section{A Partial Order on the Orthogonal Group}

\author{
Colum Watt \\ Technological University Dublin, colum.watt@TUDublin.ie \\ Thomas Brady \\ Dublin City University, thomas.brady@dcu.ie
}

Follow this and additional works at: https://arrow.tudublin.ie/scschmatart

Part of the Mathematics Commons

\section{Recommended Citation}

Watt, C., Brady, T. : A Partial Order on the Orthogonal Group. Communications in Algebra, Vol. 30, (2002), no. 8, pp 3749-3754 doi.org/10.21427/gbt1-8m22

This Article is brought to you for free and open access by the School of Mathematics at ARROW@TU Dublin. It has been accepted for inclusion in Articles by an authorized administrator of ARROW@TU Dublin. For more information, please contact arrow.admin@tudublin.ie, aisling.coyne@tudublin.ie,gerard.connolly@tudublin.ie.

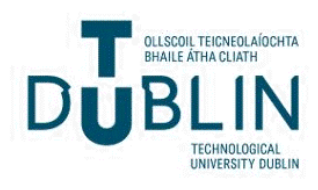




\title{
A partial order on the Orthogonal Group
}

\author{
Thomas Brady \\ School of Mathematical Sciences \\ Dublin City University \\ Glasnevin, Dublin 9 \\ Ireland \\ tom.brady@dcu.ie
}

\author{
Colum Watt \\ School of Mathematics \\ Trinity College \\ Dublin 2 \\ Ireland \\ colum@maths.tcd.ie
}

\begin{abstract}
We define a natural partial order on the orthogonal group and completely describe the intervals in this partial order. The main technical ingredient is that an orthogonal transformation induces a unique orthogonal transformation on each subspace of the orthogonal complement of its fixed subspace.
\end{abstract}

Keywords: orthogonal group, partial order.

Let $V$ be an $n$-dimensional vector space over a field $\mathbf{F}$ and let $O(V)$ be the orthogonal group of $V$ with respect to a fixed anisotropic symmetric bilinear form $\langle$,$\rangle . In this note we will define a natural partial order on O(V)$ and completely describe the intervals in this partial order. The main technical ingredient is that an orthogonal transformation $A$ on $V$ induces a unique orthogonal transformation on each subspace of the orthogonal complement of the fixed subspace of $A$.

Recall that $A \in O(V)$ if $A: V \rightarrow V$ is linear and satisfies $\langle A(\vec{v}), A(\vec{w})\rangle=$ $\langle\vec{v}, \vec{w}\rangle$ for all $\vec{v}, \vec{w} \in V$. For standard results on symmetric bilinear forms and their associated orthogonal groups see $[\mathbf{1}]$, but note that we are making the further assumption that the form is anisotropic.

For each $A \in O(V)$, we define two subspaces of $V, F(A)=\operatorname{ker}(A-I)$ and $M(A)=\operatorname{im}(A-I)$, where $I$ is the identity operator on $V$. We note that $F(A)$ is the +1 -eigenspace of $A$, sometimes called the fixed subspace of $A$. We will write $V=V_{1} \perp V_{2}$ whenever $V$ is the orthogonal direct sum of 
subspaces $V_{1}$ and $V_{2}$.

Proposition $1 V=F(A) \perp M(A)$

Proof. Since the dimensions of $F(A)$ and $M(A)$ are complementary and the form is anisotropic, it suffices to show that these subspaces are orthogonal. So let $\vec{x} \in F(A)$ and $\vec{y} \in M(A)$. Then $\vec{x}=A(\vec{x})$ and $\vec{y}=(A-I) \vec{z}$ for some $\vec{z} \in V$. Thus

$$
\langle\vec{x}, \vec{y}\rangle=\langle\vec{x},(A-I) \vec{z}\rangle=\langle\vec{x}, A(\vec{z})\rangle-\langle\vec{x}, \vec{z}\rangle=\langle A(\vec{x}), A(\vec{z})\rangle-\langle\vec{x}, \vec{z}\rangle=0 .
$$

q.e.d.

We will be concerned with how the dimensions of these subspaces behave when we take products in $O(V)$. For notational convenience we will write $|U|$ for $\operatorname{dim}(U)$.

Proposition $2|M(A B)| \leq|M(A)|+|M(B)|$ for $A, B \in O(V)$.

Proof. Using the identities $|U|+|V|=|U+V|+|U \cap V|, F(A) \cap F(B) \subseteq$ $F(A B)$ and $F(A)+F(B) \subseteq V$ we find that

$$
|F(A)|+|F(B)|=|F(A)+F(B)|+|F(A) \cap F(B)| \leq n+|F(A B)|,
$$

from which the result follows. q.e.d.

This result is proved in a more general setting in [2]. However, from the proof above we see that equality occurs if and only if

$$
F(A) \cap F(B)=F(A B) \quad \text { and } \quad F(A)+F(B)=V .
$$

Thus, using the identities $[U+V]^{\perp}=U^{\perp} \cap V^{\perp}$ and $U^{\perp}+V^{\perp}=[U \cap V]^{\perp}$ we get the following characterization.

Corollary $1|M(A B)|=|M(A)|+|M(B)| \Leftrightarrow M(A B)=M(A) \oplus M(B)$.

Definition 1 We will write $A \leq C$ if $|M(C)|=|M(A)|+\left|M\left(A^{-1} C\right)\right|$.

Proposition 3 The relation $\leq$ is a partial order on $O(V)$ and satisfies

$$
A \leq B \leq C \Rightarrow A^{-1} B \leq A^{-1} C .
$$


Proof. Reflexivity is immediate. To establish antisymmetry suppose $A \leq C$ and $C \leq A$. Then

$$
|M(C)|=|M(A)|+\left|M\left(A^{-1} C\right)\right|=|M(C)|+\left|M\left(C^{-1} A\right)\right|+\left|M\left(A^{-1} C\right)\right|
$$

giving $F\left(C^{-1} A\right)=F\left(A^{-1} C\right)=V$ or $A=C$.

To establish transitivity, suppose $A \leq B$ and $B \leq C$. Then

$$
\begin{aligned}
|M(C)| & \leq|M(A)|+\left|M\left(A^{-1} C\right)\right| \\
& =|M(A)|+\left|M\left(A^{-1} B B^{-1} C\right)\right| \\
& \leq|M(A)|+\left|M\left(A^{-1} B\right)\right|+\left|M\left(B^{-1} C\right)\right| \\
& =|M(A)|+\{|M(B)|-|M(A)|\}+\{|M(C)|-|M(B)|\} \\
& =|M(C)|
\end{aligned}
$$

So both of the inequalities are actually equalities. The first line gives $A \leq C$ and $\leq$ is transitive. The third line gives the second assertion above. q.e.d.

The association of the subspace $M(A)$ to an element $A \in O(V)$ defines a map $M$ from $O(V)$ to the set of subspaces of $V$. The next sequence of lemmas shows that the restriction of $M$ to the interval $[I, C]=\{A \in O(V) \mid A \leq C\}$ is a bijection onto the set of subspaces of $M(C)$.

In what follows we fix $C$ and a subspace $W$ of $M(C)$ and we suppose that $A \in O(V)$ satisfies $M(A)=W$. We define $U$ to be the unique subspace of $M(C)$ which satisfies $|U|=|W|$ and $(C-I) U=W$. This is possible since $C-I$ is invertible when restricted to $M(C)$.

Lemma 1 If $W \subseteq M(C)$ then $V=W^{\perp} \oplus U$.

Proof. Since the subspaces have complementary dimensions it suffices to show that their intersection is trivial. So let $\vec{x} \in W^{\perp} \cap U$. Then $\vec{x} \in W^{\perp}$ and $(C-I) \vec{x}=\vec{w}$ for some $\vec{w} \in W$. Thus $C \vec{x}=\vec{x}+\vec{w}$, with $\vec{x} \in W^{\perp}$ and $\vec{w} \in W$ so that

$$
\langle\vec{x}, \vec{x}\rangle=\langle C \vec{x}, C \vec{x}\rangle=\langle\vec{x}+\vec{w}, \vec{x}+\vec{w}\rangle=\langle\vec{x}, \vec{x}\rangle+\langle\vec{w}, \vec{w}\rangle .
$$

Thus $\vec{w}=\overrightarrow{0}$ since $\langle$,$\rangle is anisotropic and \vec{x}=\overrightarrow{0}$ since $C-I$ is an isomorphism on $M(C)$. q.e.d.

Lemma $2 F\left(A^{-1} C\right) \subseteq F(C) \perp U$. 
Proof. Let $\vec{x} \in F\left(A^{-1} C\right)$. Then $A^{-1} C \vec{x}=\vec{x}$, which implies $C \vec{x}=A \vec{x}$ and $(C-I) \vec{x}=(A-I) \vec{x}$. Using $V=F(C) \perp M(C)$ we can express $\vec{x}$ uniquely as $\vec{x}=\vec{y}+\vec{z}$ with $\vec{y} \in F(C)$ and $\vec{z} \in M(C)$. Thus

$$
(C-I) \vec{z}=(C-I) \vec{x}=(A-I) \vec{x} \in M(A)=W,
$$

giving $\vec{z} \in U$. This gives $F\left(A^{-1} C\right) \subseteq F(C)+U$ and the orthogonality of the subspaces follows since $U \subseteq M(C)$. q.e.d.

Lemma 3 If $M(A)=W$ and $A \leq C$ then $F\left(A^{-1} C\right)=F(C) \perp U$.

Proof. Since $A \leq C$ we have $\left|M\left(A^{-1} C\right)\right|=|M(C)|-|M(A)|$ so that

$$
\left|F\left(A^{-1} C\right)\right|=n-\left|M\left(A^{-1} C\right)\right|=n-|M(C)|+|W|=|F(C)|+|U| .
$$

This dimension calculation can now be combined with Lemma 2. q.e.d.

It is now possible to give a formula for $A$. If $V=V_{1} \oplus V_{2}$ we define the projection $\operatorname{Proj}_{V_{1}}^{V_{2}}$ to be the linear transformation which coincides with the identity on $V_{1}$ and with the zero transformation on $V_{2}$.

Lemma 4 If $A \leq C$ and $M(A)=W$ then $A=I+(C-I) \operatorname{Proj}_{U}^{W^{\perp}}$.

Proof. If $M(A)=W$ then $F(A)=W^{\perp}$ so that $A$ coincides with $I$ on $W^{\perp}$. Since $F\left(A^{-1} C\right)$ contains $U$ by Lemma $3, A$ coincides with $C$ on $U$. Thus $A-I$ coincides with the zero transformation on $W^{\perp}$ and with $C-I$ on $U$, giving $A-I=(C-I) \operatorname{Proj}_{U}^{W^{\perp}}$, by Lemma 1. q.e.d.

It is not at all clear from this formula that $A$ is orthogonal. However this is indeed the case.

Lemma $5 A=I+(C-I) \operatorname{Proj}_{U}^{W^{\perp}} \in O(V)$.

Proof. Let $\vec{x}, \vec{y} \in V$ and use Lemma 1 to express $\vec{x}=\vec{x}_{1}+\vec{x}_{2}, \vec{y}=\vec{y}_{1}+\vec{y}_{2}$, with $\vec{x}_{1}, \vec{y}_{1} \in U$ and $\vec{x}_{2}, \vec{y}_{2} \in W^{\perp}$. Then, using the fact that $A$ coincides with $I$ on $W^{\perp}$ and with $C$ on $U$,

$$
\begin{aligned}
\langle A(\vec{x}), A(\vec{y})\rangle & =\left\langle C\left(\vec{x}_{1}\right)+\vec{x}_{2}, C\left(\vec{y}_{1}\right)+\vec{y}_{2}\right\rangle \\
& =\left\langle C \vec{x}_{1}, C \vec{y}_{1}\right\rangle+\left\langle C \vec{x}_{1}, \vec{y}_{2}\right\rangle+\left\langle\vec{x}_{2}, C \vec{y}_{1}\right\rangle+\left\langle\vec{x}_{2}, \vec{y}_{2}\right\rangle \\
& =\left\langle\vec{x}_{1}, \vec{y}_{1}\right\rangle+\left\langle C \vec{x}_{1}, \vec{y}_{2}\right\rangle+\left\langle\vec{x}_{2}, C \vec{y}_{1}\right\rangle+\left\langle\vec{x}_{2}, \vec{y}_{2}\right\rangle \\
& =\langle\vec{x}, \vec{y}\rangle+\left\langle(C-I) \vec{x}_{1}, \vec{y}_{2}\right\rangle+\left\langle\vec{x}_{2},(C-I) \vec{y}_{1}\right\rangle \\
& =\langle\vec{x}, \vec{y}\rangle,
\end{aligned}
$$


since both $(C-I) \vec{x}_{1}$ and $(C-I) \vec{y}_{1}$ lie in $W$. q.e.d.

We will call $A$ the transformation induced by $C$ on $W$. Combining the above lemmas we get the following result.

Theorem 1 If $C \in O(V)$ and $W$ is a subspace of $M(C)$ then there exists a unique $A \in O(V)$ satisfying $A \leq C$ and $M(A)=W$.

The induced transformations are familiar objects for two special classes of subspace.

Corollary 2 If $W \subseteq M(C)$ is an invariant subspace of $C$, then the induced transformation on $W$ is the restriction of $C$ to $W$.

Proof. In this case, $U=W$ and the projection in the formula for $A$ becomes an orthogonal projection. q.e.d.

Corollary 3 If char $(\mathbf{F}) \neq 2$ and $W$ is a one dimensional subspace of $M(C)$ then the orthogonal transformation induced by $C$ on $W$ is always the orthogonal reflection in $W^{\perp}$.

Proof. Since $W$ is one-dimensional $A$ must act on $W$ by multiplication by a scalar $\alpha$. The orthogonality of $A$ forces $\alpha^{2}=1$ and $W=M(A)$ gives $\alpha \neq 1$. q.e.d.

The poset $(O(V), \leq)$ is not a lattice, since distinct elements $C_{1}$ and $C_{2}$ with $M\left(C_{1}\right)=M\left(C_{2}\right)$ cannot have a common upper bound. However the intervals are lattices and can be easily described.

Theorem 2 If $A \leq C$ in $O(V)$ and $|M(C)|-|M(A)|=m$ then the interval $[A, C]=\{B \in O(V) \mid A \leq B \leq C\}$ is isomorphic to the lattice of subspaces of $\mathbf{F}^{m}$ under inclusion.

Proof. The lattices of subspaces of $\mathbf{F}^{m}$ under inclusion is isomorphic to the interval $[M(A), M(C)]$ in the lattice of subspaces of $V$. The function $B \mapsto M(B)$ is a bijection from the interval $[A, C]$ to the latter interval by Theorem 1 . This map respects the partial orders by Corollary 1 . To see that the inverse map respects the partial orders suppose that $M(A) \subseteq$ $W_{1} \subseteq W_{2} \subseteq M(C)$. Let $B_{1}, B_{2}$ be the transformations induced on $W_{1}$, $W_{2}$ respectively by $C$ and let $B_{1}^{\prime}$ be the transformation induced on $W_{1}$ by $B_{2}$. Then $B_{1}^{\prime} \leq B_{2} \leq C$ gives $B_{1}^{\prime} \leq C$, but $M\left(B_{1}\right)=M\left(B_{1}^{\prime}\right)=W_{1}$ so the uniqueness part of Theorem 1 gives $B_{1}=B_{1}^{\prime}$ and $B_{1} \leq B_{2}$. q.e.d.

Each chain in $M(C)$ thus gives rise to a special factorization of $C$. 
Corollary 4 If $C \in O(V)$ and $W_{1} \subset W_{2} \subset \cdots \subset W_{k}=M(C)$ is a chain of subspaces in $M(C)$ then $C$ factors uniquely as a product of $k$ transformations $C=B_{1} B_{2} \ldots B_{k}$, with $B_{1} B_{2} \ldots B_{i} \leq C$ and $M\left(B_{1} B_{2} \ldots B_{i}\right)=W_{i}$.

Proof. If we define $C_{i}$ to be the transformation induced by $C$ on $W_{i}$ then $B_{i}=\left(C_{i-1}\right)^{-1} C_{i}$. q.e.d.

The case where this chain is maximal gives a strong version of the CartanDieudonné theorem.

Corollary 5 If $\operatorname{char}(\mathbf{F}) \neq 2, C \in O(V)$ with $|M(C)|=k$ and $W_{1} \subset W_{2} \subset$ $\cdots \subset W_{k}=M(C)$ is a maximal flag in $M(C)$ then $C$ factors uniquely as a product of $k$ reflections, $C=R_{1} R_{2} \ldots R_{k}$, with $M\left(R_{1} R_{2} \ldots R_{i}\right)=W_{i}$.

Proof. Here the transformation $B_{i}$ defined in Corollary 4 satisfies $\left|M\left(B_{i}\right)\right|=1$ so that $B_{i}$ is a reflection by Corollary 3. q.e.d.

Note 1 Using similar methods one can prove analogs of all the above results in the case of a unitary transformation over a finite-dimensional complex vector space. In this case we deal with complex linear subspaces, the induced transformations are unitary (and hence complex linear) and complex reflections replace the above reflections.

\section{References}

[1] N. Jacobson. Basic Algebra I, Second edition. Freeman: San Francisco, 1985.

[2] P. Scherk. On the decomposition of orthogonalities into symmetries, Proc. Amer. Math. Soc. 1950, 1, 481-491. 